\title{
FPGA implementation of trellis decoders for linear block codes
}

\author{
S. Scholl ${ }^{1}$, E. Leonardi ${ }^{2}$, and N. Wehn ${ }^{1}$ \\ ${ }^{1}$ Microelectronic Systems Design Research Group, University of Kaiserslautern, 67663 Kaiserslautern, Germany \\ ${ }^{2}$ Institute of Informatics, Federal University of Rio Grande do Sul, 91501970 Porto Alegre, Brazil
}

Correspondence to: S. Scholl (scholl@eit.uni-kl.de)

Received: 13 December 2013 - Accepted: 5 February 2014 - Published: 10 November 2014

\begin{abstract}
Forward error correction based on trellises has been widely adopted for convolutional codes. Because of their efficiency, they have also gained a lot of interest from a theoretic and algorithm point of view for the decoding of block codes. In this paper we present for the first time hardware architectures and implementations for trellis decoding of block codes. A key feature is the use of a sophisticated permutation network, the Banyan network, to implement the time varying structure of the trellis. We have implemented the Viterbi and the max-log-MAP algorithm in different folded versions on a Xilinx Virtex 6 FPGA.
\end{abstract}

\section{Introduction}

Forward error correction is widely used in today's communication systems for the correction of transmission errors. In the last years and decades many different error correction schemes have been introduced and successfully adopted in various communication standards. Prominent examples for channel codes are convolutional codes, Reed-Solomon codes, turbo codes and LDPC codes.

The optimal correction strategy is called maximum likelihood (ML) decoding. Since ML decoding is very complex for many practically used codes, most of the decoding algorithms are suboptimal heuristics, e.g. the turbo decoding algorithm (Lin and Costello, 2004). However, in this paper we consider two algorithms, that can efficiently perform ML decoding of convolutional codes or small block codes: the Viterbi algorithm (VA) (Viterbi, 1967) and the BCJR algorithm (Bahl et al., 1974).

The efficiency of the VA and BCJR algorithm originate mainly in the exploitation of the code's structure, which is graphically represented as a trellis diagram. In the past a vast amount of research has been carried out on trellis based de- coding for convolutional codes, including works from general theory to hardware implementations for real world applications.

However, not only convolutional codes can be described by trellis diagrams. Also block codes can be represented as trellis and thus efficient decoding algorithms for convolutional trellises can also be applied to block codes. Trellis decoders for block codes have many different applications as standalone ML decoder or as components of larger decoding heuristics. Here we want to point out just a few use cases:

- as a maximum likelihood decoder for small block codes

- as a component decoder for turbo product codes, Pyndiah et al. (1994)

- as a check node decoder for generalized LDPC codes, Tanner (1981)

- as a component for soft decision decoding of ReedSolomon codes, e.g. in Vardy and Be'ery (1991), Liu and Lin (2004)

Trellises of block codes mostly have a special structure (called time varying trellis), that poses a major challenge for the hardware designer. So far hardware architectures and implementations have not been considered yet. In Kim et al. (2003) a trellis decoder was implemented on an FPGA, but its use is restricted to a small group of block codes, that do not have a time varying structure.

In this paper we propose an architecture, that is able to handle all block codes of reasonable size. We solve the challenge posed by the time varying structure by introducing a optimized Banyan permutation network, that is tailored to the application. We evaluate the architectures for the VA and BCJR as well as folded versions by implementing them on 
a Virtex 6. To our best knowledge, this is the first hardware implementation of trellis decoding for arbitrary block codes.

The paper is structured as follows: in Sect. 2 we first present the construction rules for a trellis, followed by a brief description of the algorithms in Sect. 3. Section 4 describes the proposed architectures and the implementation results can be found in Sect. 5 .

\section{Trellis construction for block codes}

We consider a binary block code with block length $N$ and $K$ information bits. The code is defined by its parity check matrix $(\mathrm{PCM}) \mathbf{H}$ of dimension $(N-K) \times N$. The columns of $\mathbf{H}$ are denoted by $\mathbf{h}_{k}$. A valid code word is denoted by $\mathbf{x}=\left(x_{0}, x_{1}, \ldots, x_{N-1}\right)$ and the received log-likelihood ratios (LLRs) by $\mathbf{y}=\left(y_{0}, y_{1}, \ldots, y_{N-1}\right)$

A trellis diagram is a graphical representation of the code word space of a channel code. Every path in a trellis connecting the starting and end point correspond to exactly one code word.

The trellis diagram for block codes can be constructed in two different ways: using the generator matrix or the parity check matrix (PCM) of the code.

The first construction method is based on the generator matrix (see Lin and Costello (2004) for more information). Since this construction method is quite complex and requires the generator matrix to be in a special form (trellis oriented generator matrix form), we apply the second construction method based on the PCM.

The PCM method provides full flexibility on the matrix, so that any PCM can be used. Furthermore, the trellis structure can easily be deduced from the PCM, which makes it suitable for a hardware implementation. Since the trellis describes a block code, we call it a bit-level trellis - in contrast to the convolutional trellis. In the following we describe the construction method of Wolf (1978).

The bit-level trellis has $N$ trellis steps (sometimes called time steps), and $M=2^{N-K}$ states per step. The states are labelled by a binary $N-K$ tuple $\mathbf{s}_{k}^{m}$, where $m=0,1, \ldots, M-$ 1 denotes the index of a state in step $k=0,1, \ldots, N$.

To construct the trellis, the states between step $k$ and $k+1$ are connected by branches. There are two different types of branches. One corresponds to code bit ' 0 ' $\left(x_{k}=0\right)$ the other one to code bit ' 1 ' $\left(x_{k}=1\right)$. The branches are established recursively as follows: From each state $\mathbf{s}_{k}^{m}$ of step $k$ two branches depart to state

$\mathbf{s}_{k+1}^{m}=\mathbf{s}_{k}^{m} \quad$ for $x_{k}=0$

$\mathbf{s}_{k+1}^{l}=\mathbf{s}_{k}^{m}+\mathbf{h}_{k}$ for $x_{k}=1$

Since only paths from $\mathbf{s}_{0}^{0}$ to $\mathbf{s}_{N}^{0}$ represent code words, all other paths can be expurgated.

There are some remarkable differences between a convolutional trellis and a bit-level trellis. A bit-level trellis is in gen-

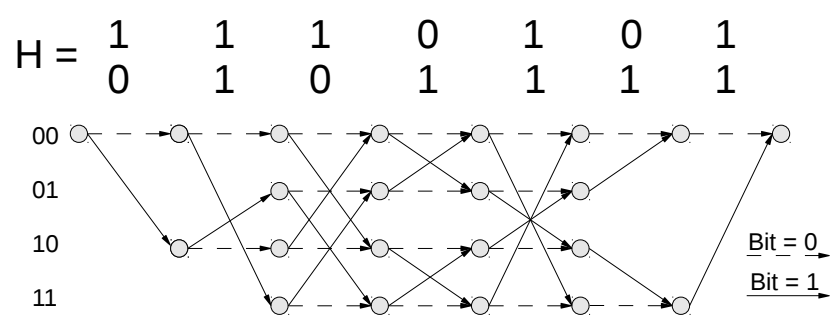

Figure 1. Example for a trellis construction using the PCM $\mathbf{H}$

eral a time-varying trellis, i.e. state transitions change over time - in contrast to the convolutional trellis.

Besides the time varying property, in a bit-level trellis only one bit is associated with a time step. In a convolutional trellis usually two or more bits correspond to one time step.

Additionally, it should be pointed out that the " 0 " branches always connect states having the same label. This property can be exploited in hardware, as we will see later.

A small example for $N=7$ and $K=5$ with a $M=4$ state trellis is depicted in Fig. 1 to clarify the trellis construction.

\section{Trellis decoding algorithms}

Once the trellis representation of a code is obtained, it enables the use of very efficient decoding algorithms, like the $\mathrm{VA}$ and the BCJR algorithm. In this section we give a short summary of these two algorithms for bit-level trellises and their variants in the log domain.

\subsection{Viterbi algorithm}

The VA (Viterbi, 1967) performs ML decoding efficiently on a trellis. It looks for the most probable path in the trellis by recursively building up paths through the trellis and discarding unlikely paths in every step. We shortly repeat the VA in the logarithm domain, that is usually used for hardware implementation.

For the VA every trellis state $\mathbf{s}_{k}^{m}$ is assigned a state metric $\alpha\left(\mathbf{s}_{k}^{m}\right)$. The state metrics for step $k$ are calculated recursively from those of step $k-1$.

1. initialize the state metrics at step $0: \mathbf{s}_{0}^{0}$ with 0 and all other $\mathbf{s}_{0}^{m}(m \neq 0)$ with -infinity

2. For all states $k=1, \ldots, N-1$ and all indices $m=$ $0,1 . ., M-1$ calculate

$\alpha\left(\mathbf{s}_{k}^{l}\right)=\max \left[\alpha\left(\mathbf{s}_{k-1}^{l}\right), \alpha\left(\mathbf{s}_{k-1}^{m}\right)+y_{k-1}\right]$

where $\mathbf{s}_{k}^{l}=\mathbf{s}_{k-1}^{m}+\mathbf{h}_{k-1}$

3. output the path which led to the maximum state metric $\alpha\left(\mathbf{s}_{N}^{0}\right)$, called the traceback step. 
More detailed information on the VA can easily be found in literature (Lin and Costello, 2004; Wolf, 1978).

\subsection{BCJR and Max-log-MAP algorithm}

A drawback of the VA is that it does not provide any soft output information, which is required by modern decoding heuristics. However, the BCJR algorithm (Bahl et al., 1974) provides such additional information.

For hardware implementations is it advantageous to use the BCJR algorithm in the logarithm domain, which is called log-MAP, or its low complexity version max-log-MAP (Robertson et al., 1995). In this paper we consider the maxlog-MAP, because it provides low complexity without degrading the decoding performance significantly.

In the max-log-MAP (and also the BCJR algorithm) every state is assigned two state metrics: the forward state metrics $\alpha\left(\mathbf{s}_{k}^{m}\right)$ and the backward state metrics $\beta\left(\mathbf{s}_{k}^{m}\right)$.

Max-log-MAP decoding consists of three phases:

1. forward recursion (calculates state metrics $\alpha\left(\mathbf{s}_{k}^{m}\right)$ )

2. backward recursion (calculates state metrics $\left(\beta\left(\mathbf{s}_{k}^{m}\right)\right)$

3. soft output calculation (using $\alpha\left(\mathbf{s}_{k}^{m}\right), \beta\left(\mathbf{s}_{k+1}^{m}\right)$ and $\left.y_{k}\right)$

The forward recursion is equal to that of the VA in Eq. (1). For the backward recursion the states are processed in reversed order, i.e. from right to left. Details of the algorithm can be found e.g. in Lin and Costello (2004).

It can already be seen, that the recursion steps are important in both the VA and the max-log-MAP. It consists of add-compare-select (ACS) operations in Eq. (1) and requires permutations of state metrics according to the time varying branch structure. In the following section, we will focus on the hardware architecture for such a recursion unit for bitlevel trellises.

\section{Proposed architectures}

In this section we present the hardware architectures for the VA and the max-log-MAP decoder for bit-level trellises. We propose the use of a Banyan network to implement the flexible state transitions. Finally we present a folded architecture, which reduces the decoder area to enable the implementation for trellises with a large number of states.

\subsection{Architectures for Viterbi and Max-Log-MAP algorithm}

The top level architectures for Viterbi and max-log-MAP decoder are depicted in Figs. 2 and 3. Although they look different, their core functionality is the recursion unit, which is identical in both cases.

The recursion unit for a simple case of a 4 state trellis is shown in Fig. 4. All state metrics $\alpha\left(\mathbf{s}_{k}^{m}\right)$ of one time step $k$ are

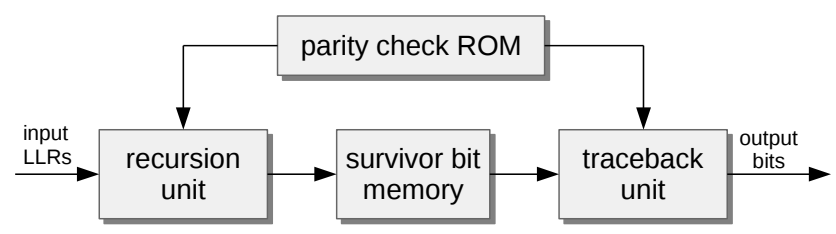

Figure 2. Top level architecture of the Viterbi decoder

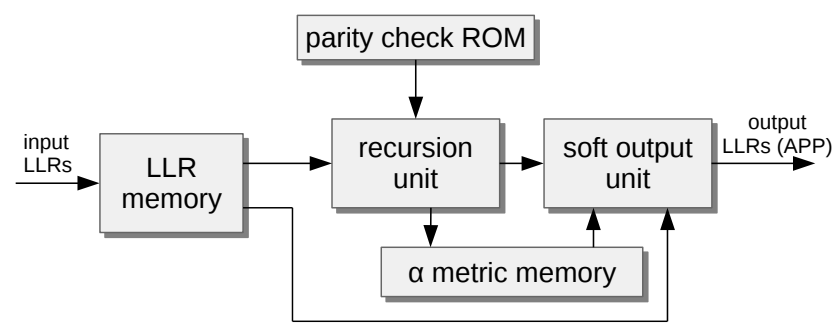

Figure 3. Top level architecture for the max-log-MAP decoder

calculated in parallel. The different trellis steps are calculated consecutively, i.e. one trellis step per clock cycle. The state metrics are temporarily stored in the registers.

One iteration consists of routing the state metrics through the permutation network first. The routing is done according to the branch structure of the trellis and is determined by the columns of the PCM $\mathbf{h}_{k}$. Then the ACS units calculate Eq. (1). Finally the state metrics are stored in the register again.

Note, that the state metrics are directly fed back to the ACS units. This maps the branches for code bit ' 0 ' to the hardware.

The state metrics and received LLRs $\mathbf{y}$ are represented as fixed point numbers. To keep the number of bits low and therefore to save resources without provoking catastrophic overflows, modulo arithmetic is used here (Hekstra, 1989).

\subsection{Permutation network}

An essential part of the trellis decoders is the permutation network (PN). It routes the state metrics along the Bit " 1 " branches during the recursion.

The PNs in the trellis decoders must have $M=2^{N-K}$ inputs and outputs. The network is controlled by the column bits of the PCM $\mathbf{h}_{k}$ and consists of $2 \times 2$ butterfly switches, which can permute or route through its two inputs.

We first investigate the use of the well known Benes PN (Benes, 1964) and in a second step propose the use of a more sophisticated network, the Banyan PN (Goke and Lipovski, 1973).

The Benes PN is capable of performing all possible $M$ ! permutations, although in this application only $M$ are required. Therefore, the Benes PN uses more resources than necessary. A drawback of the Benes PN is its elevated number of $2 \cdot \log _{2}(M)-1$ stages, which directly lengthens 


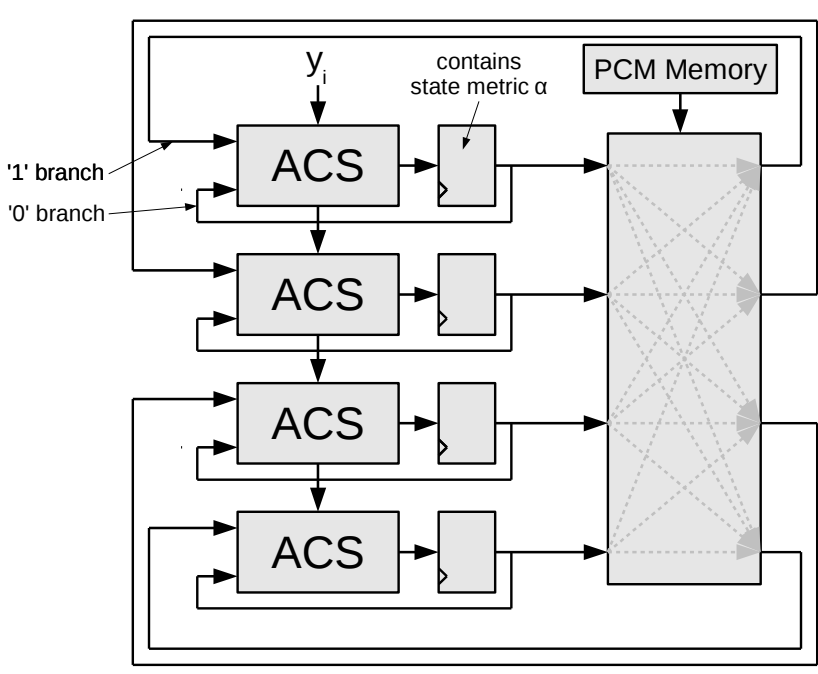

Figure 4. Architecture of the recursion unit

the maximum combinational path of the recursion unit and thus decreases the throughput. Furthermore the Benes PN requires a complex controlling logic, which maps the bits from the PCM to the control bits of the switches, see Fig. 7.

The second network studied is the Banyan PN. It performs the necessary permutation with approximately half the number of stages $\left(\log _{2}(M)\right)$ in comparison to the Benes PN. This reduces the signal propagation time and improves throughput. Moreover the Banyan PN has approximately half the number of switches (see Fig. 5). Another advantage is that the bits of the columns of the PCM can directly be applied to control the switches in the network. No extra controlling logic is required. The small number of switches and the saving of the complex controlling logic makes the Banyan PN the network of choice for this application.

In the following, we provide our method of how to build up such a network. The Banyan PN is constructed from two Butterfly PNs and a final permutation stage. The smallest Butterfly PN is composed of a single switch and larger ones can be constructed recursively, i.e. a network with $M$ inputs is obtained from two Butterfly PNs with $M / 2$ inputs.

We place the second Butterfly PN below the first one and denote the outputs by $S(i)$ with $i=0,1, . ., M / 2-1$ and $i=$ $M / 2, . ., M-1$. The final stage is a column of $M / 2$ switches that placed right of the two Butterfly PNs. Their inputs are denoted by $I(i)$ with $i=0,1, \ldots, M-1$ and their outputs by $B(i)$. The connections between the two Butterfly PNs and the final stage is done by the following algorithm:

Finally, we connect the outputs $B(i)$ of the final stage to the outputs $O(i)$ of the Banyan PN by using the following algorithm:

Each input of the network has to be able to reach all the $M$ different output addresses and that is only possible with $N-K$ stages. Thus it is impossible to do all the required permutations with a network with less stages than the Banyan.
Algorithm 1. Intermediate connections of a Butterfly PN.

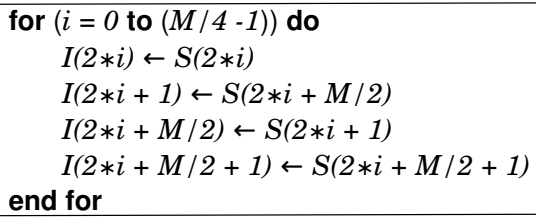

Algorithm 2. Output connections of a Banyan PN.

for $(i=0$ to $(M / 2-1))$ do
$\quad O(i) \leftarrow B(2 * i)$
$\quad O(i+M / 2) \leftarrow B(2 * i+1)$
end for

Figure 6 shows the construction of an $8 \times 8$ Banyan PN using the algorithms from above.

\subsection{Folding}

For trellises with a large number of states $M$, the above presented architecture grows quickly and may become too large for an FPGA implementation. To counteract this problem, we propose folding to reduce the required resources of the decoder at the expense of a reduced throughput.

In the folded architecture not all $M$ state metrics in one trellis step are calculated in one clock cycle. Instead the state metrics are calculated, e.g. in two clock cycles. In this case half of the ACS units can be reused.

Furthermore it reduces the size of the PN by more than half given that its construction is recursive (see above). Note, that the folded Banyan PN with $M / 2$ exactly fulfils the permutation requirements of the folded architecture, and is thus suitable for folded architectures.

The number of clock cycles required to calculate the state metrics of one trellis step is called folding factor $f$ and must be a power of 2 .

Due to folding the size of the PN, the number of ACS units and the soft output unit (for the max-log-MAP decoder) is largely reduced. However, some additional hardware resources are needed to distribute the state metrics to the PN and ACS units and registers to store the partially calculated state metrics.

The area reduction of the soft output unit is directly proportional to the folding factor $f$. Therefore folding is especially advantageous for the max-log-MAP decoder.

\section{FPGA implementation}

To evaluate the architectures in detail we have implemented the architectures on a Xilinx Virtex 6 (XC6VLX75T-3) FPGA using ISE 14.1. The architectures for the VA, the max-log-MAP and their folded version have been evaluated for trellises with different number of states. We analyse the 


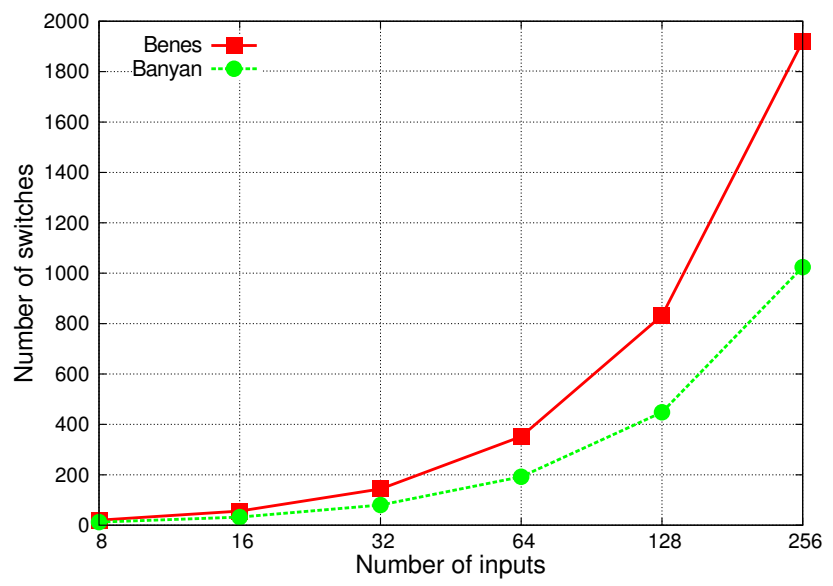

Figure 5. Number of switches used by the Benes and the Banyan PN

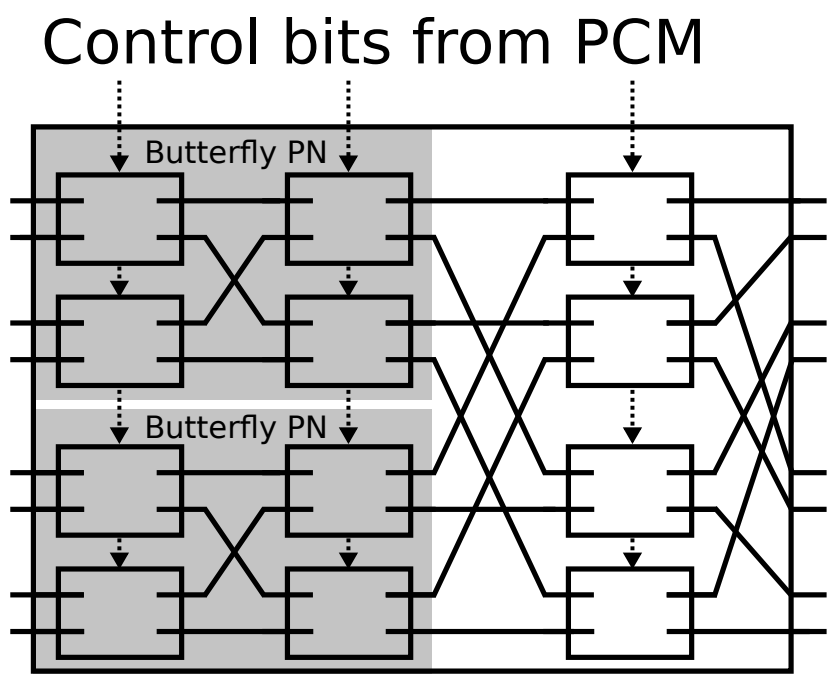

Figure 6. Eight input Banyan PN: it needs less switches than the Benes PN and no controlling is needed

resource consumption regarding the required look-up tables (LUTs) after place \& route.

The numbers presented are only dependent on the number of trellis states $M$ and not on the number of trellis steps $N$. The number of trellis steps $N$ only influences the size of the memories. However this is not the critical resource in the design.

The quantization of the metrics is dependent on the context in which the decoders are used. However it has been experienced by simulations that a state metric quantization of 7 Bits for the VA and 8 bits for the max-log-MAP is reasonable.

In Figs. 8 and 9 the required LUTs for the unfolded architecture $(f=1)$ is shown, separated for each decoder part.

For the VA almost $50 \%$ of the LUTs are occupied by the ACS units. The PN also consumes nearly $50 \%$ of the LUTs. Therefore the ACS units and the PN are the dominating parts.

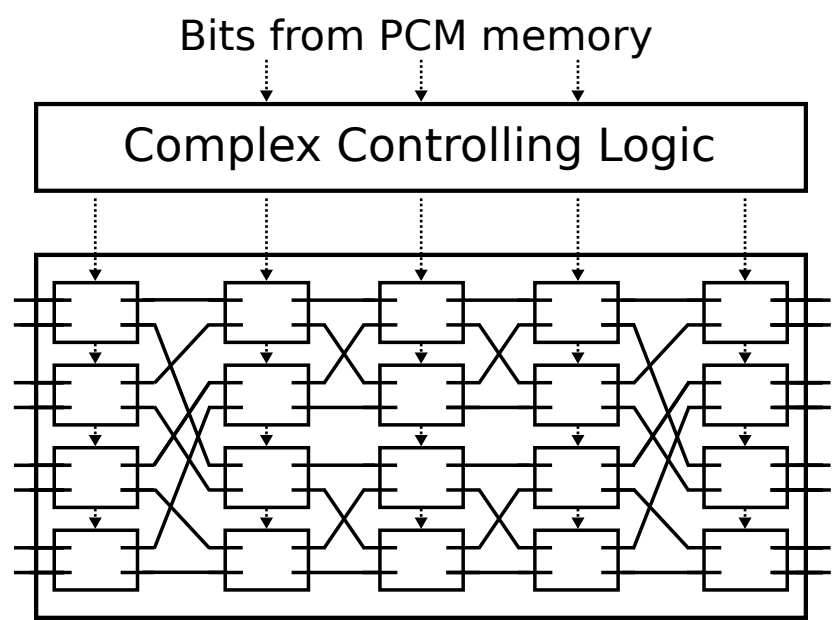

Figure 7. Eight input Benes PN: it needs more switches than the Banyan PN and additional controlling is needed

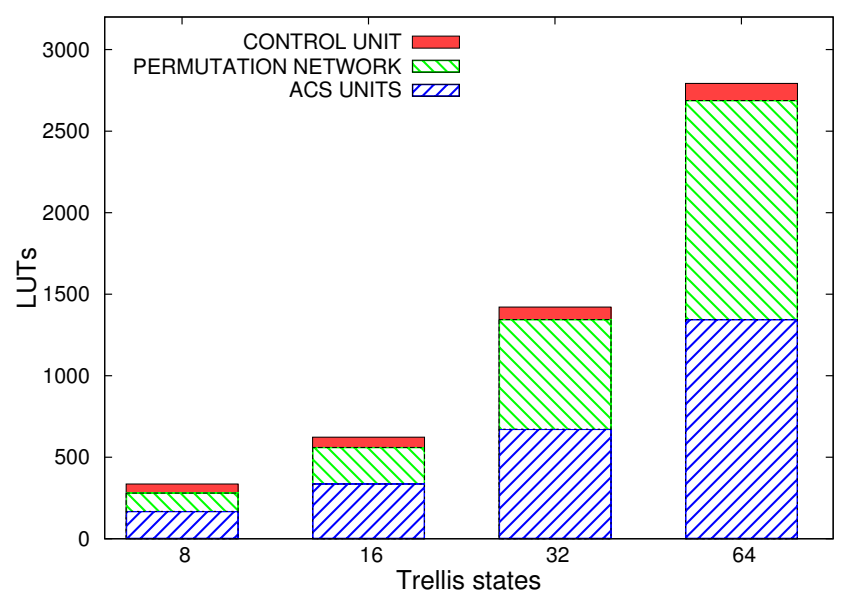

Figure 8. Viterbi decoder: look-up tables

The remaining fraction is occupied by the controlling, which also includes the traceback unit.

The max-log-MAP is dominated by the soft output unit, which needs approximately $50 \%$ of all LUTs. The PN and the ACS units consume around $25 \%$ each.

In Figs. 10 and 11 the number of required LUTs for different folded architectures are shown. It shows how the occupied resources reduce and allows for the implementation of larger trellises. The throughput decrease for the VA can be seen in Fig. 12.

\section{Conclusions}

In this paper we have investigated hardware architectures for bit-level trellises. We have selected the trellis construction based on the PCM, because it provides maximum flexibility. After a brief review of the VA and the BCJR decoding algorithms, we proposed efficient hardware architectures. A key 


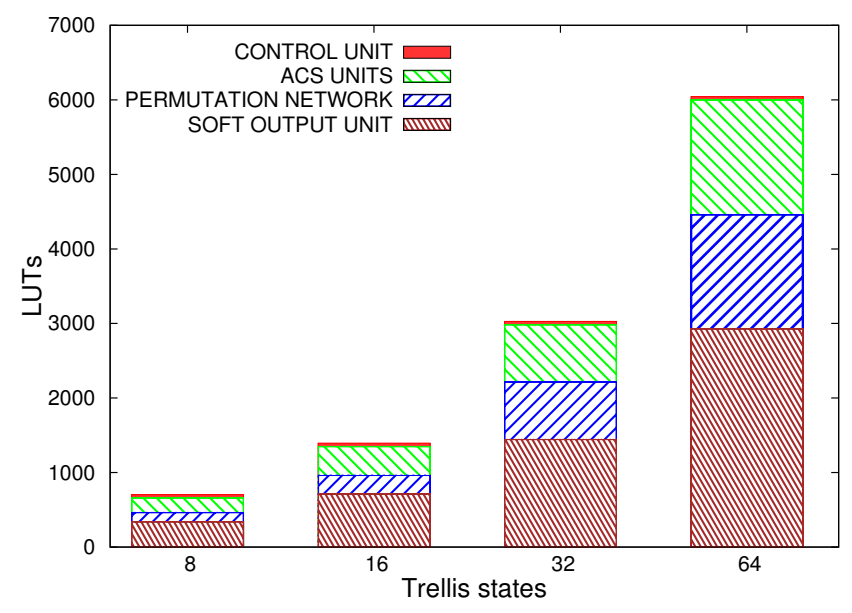

Figure 9. Max-log-MAP decoder : look-up tables

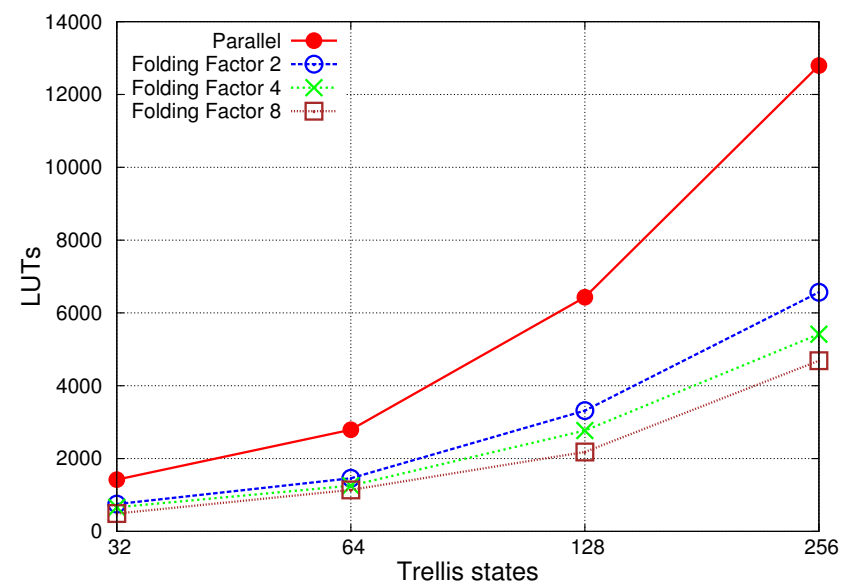

Figure 10. Viterbi decoder: LUTs dependent on folding factor

feature is the Banyan PN, which maps the time variant property of the trellis efficiently to hardware and significantly outperforms standard solutions like the Benes PN. Furthermore we presented a folded version of the architecture to enable the implementation for large trellises. Finally the resource consumption and throughput of the architecture have been evaluated on a Xilinx Virtex 6 FPGA.

Acknowledgements. We gratefully acknowledge partially financial support by the DFG (project-ID: KI 1754/1-1) as well as by the Center of Mathematical and Computational Modelling of the University of Kaiserslautern. We thank F. Kienle for his valuable comments and suggestions.

Edited by: J. Götze

Reviewed by: two anonymous referees

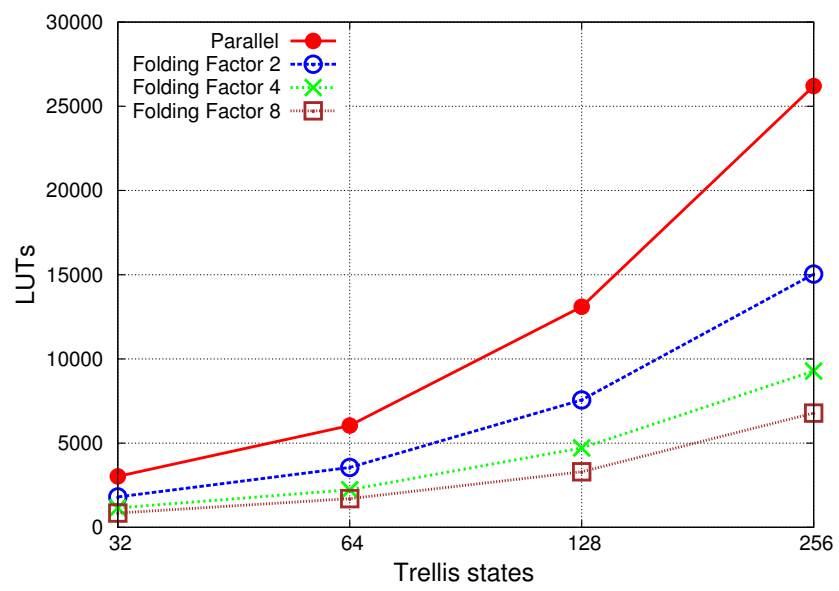

Figure 11. Max-log-MAP decoder: LUTs dependent on folding factor

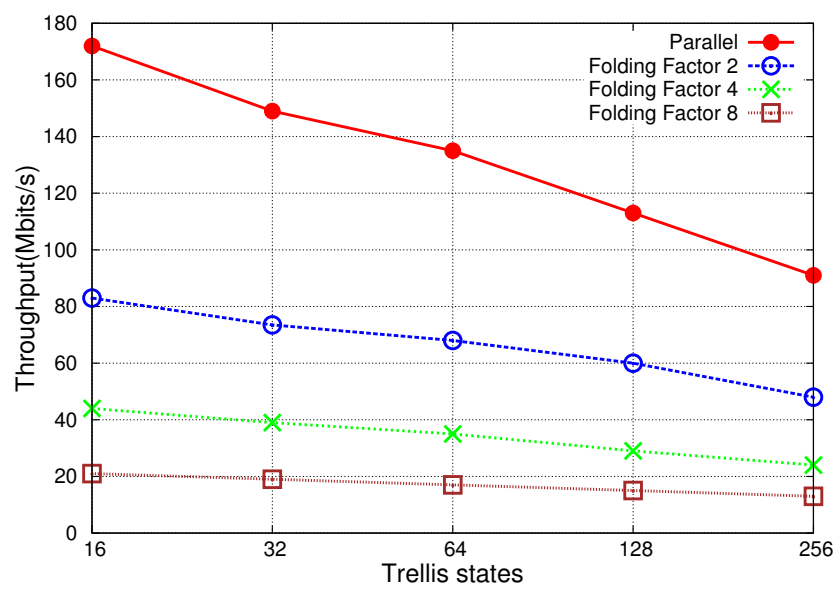

Figure 12. Viterbi decoder: throughput dependent on folding factor

\section{References}

Bahl, L., Cocke, J., Jelinek, F., and Raviv, J.: Optimal Decoding of Linear Codes for Minimizing Symbol Error Rate, IEEE T. Inform. Theory, IT-20, 284-287, 1974.

Benes, V. E.: Optimal Rearrangeable Multistage Connecting Networks, The Bell System Technical Journal, 4, 1641-1656, 1964.

Goke, L. R. and Lipovski, G. J.: Banyan Networks for Partitioning Multiprocessor Systems, 1st Annual Symposium on Computer Architecture, 21-28, 1973.

Hekstra, A. P.: An Alternative to Metric Rescaling in Viterbi Decoders, IEEE T. Commun., 37, 1220-1222, 1989.

Kim, S., Ryoo, S., and Lee, S.: Block Turbo Codes Using Multiple Soft Outputs, in: Proceedings of the 3rd ISTC, Vol. 1, 247-250, Brest, 2003.

Lin, S. and Costello, D.: Error Control Coding 2nd., Prentice Hall PTR, Upper Saddle River, New Jersey, USA, 2004.

Liu, C. Y. and Lin, S.: Turbo encoding and decoding of Reed-Solomon codes through binary decomposition and selfconcatenation, IEEE T. Commun., 52, 1484-1493, 2004. 
Pyndiah, R., Glavieux, A., Picart, A., and Jacq, S.: Near optimum decoding of product codes, in: Proc. IEEE Global Telecommunications Conf. GLOBECOM '94. Communications: The Global Bridge, 339-343, 1994.

Robertson, P., Villebrun, E., and Hoeher, P.: A Comparison of Optimal and Sub-Optimal MAP decoding Algorithms Operating in the Log-Domain, in: Proc. 1995 International Conference on Communications (ICC '95), 1009-1013, Seattle, Washington, USA, 1995.
Tanner, R. M.: A Recursive Approach to Low Complexity Codes, IEEE T. Inform. Theory, IT-27, 533-547, 1981.

Vardy, A. and Be'ery, Y.: Bit-level soft-decision decoding of ReedSolomon codes, IEEE T. Commun., 39, 440-444, 1991.

Viterbi, A. J.: Error Bounds for Convolutional Codes and an Asymptotically Optimum Decoding Algorithm, IEEE T. Inform. Theory, 13, 260-269, 1967.

Wolf, J.: Efficient maximum likelihood decoding of linear block codes using a trellis, IEEE T. Inform. Theory, 24, 76-80, 1978. 